\title{
A New Characterization of Half-Flat Solutions to Einstein's Equation
}

\author{
Abhay Ashtekar ${ }^{1,2}$, Ted Jacobson ${ }^{3,4}$, and Lee Smolin ${ }^{2,5}$ \\ ${ }^{1}$ Physics Department, Syracuse University, Syracuse, NY 13244-1130, USA \\ ${ }^{2}$ Institute for Theoretical Physics, University of California, Santa Barbara, CA 93106, USA \\ ${ }^{3}$ Physics Department, Brandeis University, Waltham, MA 02254, USA \\ ${ }^{4}$ Physics Department, University of California, Santa Barbara, CA 93106, USA \\ ${ }^{5}$ Physics Department, Yale University, New Haven, CT 06511, USA
}

\begin{abstract}
A $3+1$ formulation of complex Einstein's equation is first obtained on a real 4-manifold $M$, topologically $\Sigma \times R$, where $\Sigma$ is an arbitrary 3-manifold. The resulting constraint and evolution equations are then simplified by using variables that capture the (anti-) self dual part of the 4-dimensional Weyl curvature. As a result, to obtain a vacuum self-dual solution, one has just to solve one constraint and one "evolution" equation on a field of triads on $\Sigma$ :

$$
\operatorname{Div} V_{\mathbf{i}}^{a}=0 \text { and } \dot{V}_{\mathbf{i}}^{a}=\varepsilon_{\mathbf{i j k}}\left[V_{\mathbf{j}}, V_{\mathbf{k}}\right]^{a} \text {, with } \mathbf{i} \equiv 1,2,3 \text {, }
$$

where Div denotes divergence with respect to a fixed, non-dynamical volume element. If the triad is real, the resulting self-dual metric is real and positive definite. This characterization of self-dual solutions in terms of triads appears to be particularly well suited for analysing the issues of exact integrability of the (anti-) self-dual Einstein system. Finally, although the use of a $3+1$ decomposition seems artificial from a strict mathematical viewpoint, as David C. Robinson has recently shown, the resulting triad description is closely related to the hyperkähler geometry that (anti-) self-dual vacuum solutions naturally admit.
\end{abstract}

\section{Introduction}

Over the past decade, considerable work has been done on half-flat ${ }^{1}$ solutions to Einstein equations both in the Euclidean and the complex regimes. It turns out that the half-flatness requirement simplifies Einstein's equation significantly, whence it is possible to obtain several interesting results. The most powerful ones are the following. First, using three different approaches, Newman, Penrose, and Pleban-

\footnotetext{
${ }^{1}$ A 4-metric $g_{a b}$ will be said to be half-flat if its Riemann tensor is proportional to its dual. Note that, due to the Bianchi identity ${ }^{4} R_{[a b c] d}=0$, a half-flat metric is necessarily Ricci flat. Because the square of the duality operator equals +1 if $g_{a b}$ is Euclidean, and -1 if it is Lorentzian, a real metric can be half-flat only if it has Euclidean signature. In this case, we shall say that the metric is self dual if its Riemann tensor equals its dual and anti-self dual if it equals minus its dual
} 
ski have given algorithms to find the "general" half-flat solution. (For a review, see, e.g., [1].) The task of finding such an algorithm in the non half-flat context, on the other hand, remains hopelessly difficult in spite of the fact that the problem has drawn attention over several decades. The second striking result is on the asymptotic behavior of these solutions: Using their $H$-space techniques, Newman and collaborators have shown that, in the exact theory, the characteristic data of a self-dual solution at past null infinity are related to those at future null infinity in exactly the same way as in the linearized theory. Thus, in spite of non-linearities, the classical $S$-matrix is trivial in the half-flat case.

These and other results have led to certain conjectures concerning the mathematical structure and the physical relevance of these solutions. The results on trivial scattering suggest, for example, that the half-flat Einstein system may be exactly integrable. If this turns out to the case, it would be of considerable interest to find the associated infinite number of conservation laws and to attempt nonperturbative quantization of the resulting Hamiltonian framework. Such a quantum theory would provide a rare example of an exactly soluble model in 4-dimensional quantum field theory. Moreover, it may provide useful hints for full quantum gravity. In particular, it could shed light on the viability of Penrose's suggestion [3] that (positive frequency) half-flat solutions should be regarded as the quantum mechanical wave functions of non-linear- or "dressed"-gravitons.

Although these ideas are attractive, they have not been pursued vigorously because the techniques that have been used to construct and analyze the properties of these solutions are very different from those needed to test exact integrability and to carry out quantization. For example, although the $H$-space and twistor methods developed by Newman and Penrose are powerful in many respects, they are not geared to obtain a Hamiltonian description of half-flat fields because they involve constructions which are essentially non-local in terms of space-time variables.

The purpose of this paper is to present an alternate treatment of half-flat metrics; a treatment that is closer in spirit to the traditional Hamiltonian formulation of field theories. It is hoped that this analysis will serve as a useful platform from which one can explore the field theoretical properties of the half-flat system using conventional methods. In addition, since the final picture that emerges from our approach emphasizes the hyperkähler structures that naturally exist on half-flat space-times, the approach may have purely mathematical applications, e.g., in finding suitable generalizations of the hyperkähler structures to higher dimensions and/or to quaternionic manifolds which correspond to Einstein- rather than Ricci flat-spaces.

The plan of this paper is as follows. Section 2 is devoted to mathematical preliminaries. In Sect. 2.A, we briefly recall the standard $3+1$ decomposition of Einstein's vacuum equation for a complex metric on a real 4-manifold $M$, topologically $\Sigma \times R$ for some 3-manifold $\Sigma$, in terms of the first and second fundamental forms of $\Sigma$. Section 2 .B recalls the spin-connections, first introduced by Sen [4], which are potentials for half (i.e. for self dual or anti-self dual parts) of the Weyl curvature [5]. In Sect. 3, we introduce new variables - which are to replace the first and the second fundamental forms - and recast various projections of Einstein's equation in terms of them. The new variables consist of a pair, 
$\left(\tilde{\sigma}^{a}{ }_{A}{ }^{B},{ }^{+} A_{a A}{ }^{B}\right)$ [or, alternatively, $\left.\left(\tilde{\sigma}^{a}{ }_{A}{ }^{B},{ }^{-} A_{a A}{ }^{B}\right)\right]$, where $\tilde{\sigma}^{a}{ }_{A}{ }^{B}$ is a (density weighted) soldering form for $S L(2, C)$ - or rather, complexified $S U(2)$ - spinors and ${ }^{ \pm} A_{a A}{ }^{B}$ are $S L(2, C)$-valued Sen connections 1-forms defined on $\Sigma$. (Throughout, \pm will stand for plus or minus.) The transition from the first and second fundamental forms of $\Sigma$ to $\left(\tilde{\sigma}^{a}{ }_{A}{ }^{B},{ }^{ \pm} A_{a A}{ }^{B}\right)$ simplifies the constraint as well as the evolution equations considerably. Up to this point, the material is only a generalization of a recently introduced phase space formulation of real, Lorentzian general relativity in terms of spinorial variables [6] (except that the conventions are so fixed that when all fields under consideration are restricted to take values in the $S U(2)$ subalgebra of $S L(2, C)$-i.e. to the "real part" of the complexified $S U(2)$ - we obtain an Euclidean, rather than Lorentzian, 4-metric.) The key observation of the paper is made in Sect. 4. Since ${ }^{-} A_{a A}{ }^{B}$ is a potential for the self dual part of the Weyl tensor and ${ }^{+} A_{a A}{ }^{B}$, for the anti-self dual part, self dual solutions can be obtained by setting ${ }^{+} A_{a A}{ }^{B}$ equal to zero and anti-self dual ones, by setting ${ }^{-} A_{a A}{ }^{B}$ equal to zero. When this is done, a drastic simplification occurs: we are left with just one (linear) constraint equation and one (non-linear) "evolution equation" for the remaining dynamical variable $\tilde{\sigma}^{a}{ }_{A}{ }^{B}$. By using a triad $V_{i}^{a}$ of vectors on $\Sigma$ and Pauli matrices to express the soldering form, these equations reduce to:

$$
\begin{gathered}
\text { (Div) } V_{\mathbf{i}}^{a}=0, \quad \mathbf{i}=1,2,3 \text { and, } \\
\dot{V}^{a}{ }_{\mathbf{i}}=\varepsilon_{\mathbf{i j k}}\left[V_{\mathbf{j}}, V_{\mathbf{k}}\right]^{a} ;
\end{gathered}
$$

where the divergence in the first equation is taken with respect to a fixed, nondynamical volume element on $\Sigma$ (whose choice is intertwined with that of the lapse function), and the dot and the bracket in the second equation denote, respectively, "time-derivative" and Lie-bracket. The required half-flat 4-metric $g_{a b}$ on $M=\Sigma \times R$ can be constructed from the solution to (1) and (2) by purely algebraic manipulations. The converse is also true: every half-flat metric $g_{a b}$ on $M=\Sigma \times R$ admits, at least locally, a triad $V^{a}{ }_{i}$ satisfying (1) and (2). In this sense, (1) and (2) capture the entire content of the half-flat equation, thereby providing a new characterization of half-flat 4-metrics. To illustrate the simplicity of these equations, in Sect. 5.A we obtain a class of half-flat 4-metrics by solving (1) and (2). In Sect. 5.B we linearize these equations and recover all the right circularly polarized weak gravitational waves. Section 6 discusses possible applications of our characterization of half-flat metrics.

Our conventions are as follows. Throughout, we use Penrose's abstract index notation [7]. However, to avoid possible confusion, 4-dimensional objects will carry a prefix ${ }^{4}$ when the four dimensional and three dimensional objects are denoted by the same letter. $g_{a b}$ is assumed to be Riemannian when real and sign conventions are adapted to this case. The curvature tensors are defined by:

$$
2^{4} D_{[a},{ }^{4} D_{b]} k_{c}=:{ }^{4} R_{a b c}{ }^{d} k_{d}, \quad{ }^{4} R_{a m b}{ }^{m}=:{ }^{4} R_{a b}
$$

and

$$
{ }^{4} R_{a b} g^{a b}=:{ }^{4} R .
$$

While dealing with spinors, $\varepsilon^{A B}$ is fixed once and for all, independently of the metric; the soldering forms, $\sigma^{a}{ }_{A A^{\prime}}$, carry all the information pertaining to a specific $g_{a b}$. 


\section{Mathematical Preliminaries}

This section is divided into two parts. In the first, we recall the standard $3+1$ decomposition of the complex Einstein equation on a real 4-manifold and in the second we summarize a few properties of certain spin connections that will play an important role in Sect. 3.

\section{A The Standard 3+1 Decomposition}

Since it is somewhat unusual to consider complex solutions of Einstein's equation on real manifolds, we shall briefly sketch the $3+1$ decomposition procedure for such solutions to ensure that the entire procedure is meaningful beyond the familiar Lorentzian context.

Fix a real 3-manifold $\Sigma$ and let $M$ be the 4-manifold $\Sigma \times R$. Thus, $M$ is endowed with a natural foliation. Let the leaves of the foliation be labelled by constant values of a real coordinate $t$. (It is convenient to think of $t$ as a "time coordinate" even though we are not working with real Lorentzian metrics.) Let $g_{a b}$ be a complex metric - i.e., a second-rank, symmetric, non-degenerate complex tensor field - on $M$. (If it happens to be real, we assume it has signature ++++ and our sign conventions are adapted to this signature.) By the usual analytic argument, it follows that there is a unique torsion-free connection ${ }^{4} D$ compatible with $g_{a b}$. Hence, the Riemann, the Ricci, and the Einstein tensors of $g_{a b}$ are well-defined on $M$.

Let $\zeta^{a}$ be a smooth vector field on $M$ such that $\zeta_{a}:=g_{a b} \zeta^{b}$ is everywhere proportional to $d t$ and such that $\zeta^{a} \zeta_{a}=1$. Thus, $\zeta^{a}$ is the unit normal to $\Sigma$, unique up to an overall sign. The intrinsic metric $q_{a b}$ on a $t=$ constant slice is given by

$$
q_{a b}=g_{a b}-\zeta_{a} \zeta_{b}
$$

and the "extrinsic curvature" of this slice is

$$
K_{a b}=q_{a}{ }^{m} q_{b}{ }^{n 4} D_{m} \zeta_{n},
$$

where $q_{a}{ }^{m}$ is the projection operator associated with the slice. A straightforward calculation leads us to the Gauss-Codazzi equations relating the curvature of $g_{a b}$ with $K_{a b}$ and the (intrinsic) curvature of $q_{a b}$. One has:

$$
-2{ }^{4} G_{a b} \zeta^{a} \zeta^{b}=R+K^{a b} K_{a b}-K^{2}
$$

and

$$
G_{b c} \zeta^{b} q_{a}^{c}=D^{b}\left(K_{a b}-K q_{a b}\right),
$$

where ${ }^{4} G_{a b}=\left({ }^{4} R_{a b}-\frac{1}{2}{ }^{4} R g_{a b}\right)$ is the Einstein tensor of $g_{a b}$ and where $D$ and $R$ are, respectively, the derivative operator and the Ricci scalar of $q_{a b}$.

Set $t^{a}=\left(\mathscr{L}_{\zeta} t\right)^{-1} \zeta^{a} \equiv N \zeta^{a}$. Then $t$ is an affine parameter of the vector field $t^{a}$. It is easy to show that the "time-derivatives" of $q_{a b}$ and $K_{a b}$ are given by:

$$
\dot{q}_{a b}:=\mathscr{L}_{t} q_{a b}=2 N K_{a b} ;
$$

and

$$
\begin{aligned}
\dot{K}_{a b}:=\mathscr{L}_{t} K_{a b}= & -D_{a} D_{b} N+N R_{a b}+2 N K_{a}{ }^{m} K_{m b}-N K K_{a b} \\
& -N q_{a}{ }^{m} q_{b}{ }^{n} R_{m n},
\end{aligned}
$$


where $R_{a b}$ and ${ }^{4} R_{a b}$ are the Ricci tensors of $q_{a b}$ and $g_{a b}$ respectively. Thus, $g_{a b}$ satisfies the Einstein vacuum equation, ${ }^{4} G_{a b}=0$, if and only if the fields $q_{a b}(t)$ and $K_{a b}(t)$ satisfy, on each $t=$ constant slice, the constraint equations

$$
\begin{gathered}
R+K^{a b} K_{a b}-K^{2}=0, \quad \text { and }, \\
D^{a}\left(K_{a b}-K q_{a b}\right)=0,
\end{gathered}
$$

and the "evolution equations"

$$
\dot{q}_{a b}=2 N K_{a b},
$$

and

$$
\dot{K}_{a b}=-D_{a} D_{b} N+N R_{a b}+2 N K_{a}^{m} K_{b m}-N K K_{a b} .
$$

Thus, even though we are working with complex metrics $g_{a b}$, the $3+1$ decomposition $\left(5^{\prime}\right)-\left(8^{\prime}\right)$ of Einstein's equation is completely analogous to the real Lorentzian case. (We have set shift equal to zero just for simplicity.) Note, however, that, in the complex (or real Euclidean) case, the Cauchy problem need not be well-posed -i.e., a pair $\left(q_{a b}, K_{a b}\right)$ on $\sum$ satisfying $\left(5^{\prime}\right)$ and $\left(6^{\prime}\right)$ need not give rise to a Ricci-flat $g_{a b}$ on $\Sigma \times I$, no matter how small the real interval $I$ is - unless we further restrict $\left(q_{a b}, K_{a b}\right)$ to be, say, analytic.

\section{B The Sen Connections}

A natural framework for analysing half-flat solutions is provided by spinors. Let $g_{a b}$ be a complex metric on $M$ and let $\sigma_{a}{ }^{A A^{\prime}}$ be an $S L(2, C)$-spin soldering form compatible with $g_{a b}$ :

$$
g_{a b}=\sigma_{a}^{A A^{\prime}} \sigma_{b}^{B B^{\prime}} \varepsilon_{A B^{-}{ }_{A^{\prime} B^{\prime}}} .
$$

(Note that, because $g_{a b}$ is complex, the primed and unprimed indices are completely independent.) Let ${ }^{4} \mathscr{D}$ be the torsion-free connection on spinors and tensors, which annihilates $\sigma_{a}{ }^{A A^{\prime}}, \varepsilon_{A B}$, and $\varepsilon_{A^{\prime} B^{\prime}}$. Thus, ${ }^{4} \mathscr{D}$ is an extension to spinors of the metric compatible connection ${ }^{4} D$. Denote the curvature 2 -forms of ${ }^{4} \mathscr{D}$, by ${ }^{4} F_{a b M}{ }^{N}$ and ${ }^{4} \widetilde{F}_{a b M^{\prime}}{ }^{N^{\prime}}$. If $g_{a b}$ is Ricci-flat, these 2 -forms can be expressed in terms of the Weyl curvature of $g_{a b}[7]$ :

and

$$
\begin{aligned}
{ }^{4} F_{a b M^{\prime}}{ }^{N^{\prime}} \lambda_{N}: & =2^{4} \mathscr{D}_{[a}{ }^{4} \mathscr{D}_{b]} \lambda_{M} \\
& =\left(\sigma_{[a}{ }^{A A^{\prime}} \sigma_{b]}{ }^{B B^{\prime}} \Psi_{A B M}{ }^{N} \varepsilon_{A^{\prime} B^{\prime}}\right) \lambda_{N},
\end{aligned}
$$

$$
\begin{aligned}
{ }^{4} \widetilde{F}_{a b M^{\prime}}{ }^{N^{\prime}} \alpha_{N^{\prime}}: & =2^{4} \mathscr{D}_{[a}{ }^{4} \mathscr{D}_{b]} \alpha_{M^{\prime}} \\
& =\left(\sigma_{[a}{ }^{A A^{\prime}} \sigma_{b]}{ }^{B B^{\prime}} \widetilde{\Psi}_{A^{\prime} B^{\prime} M^{\prime}}{ }^{N^{\prime}} \varepsilon_{A B}\right) \alpha_{N^{\prime}},
\end{aligned}
$$

where $\Psi_{A B M N}$ is the anti-self dual Weyl spinor and $\widetilde{\Psi}_{A^{\prime} B^{\prime} M^{\prime} N^{\prime}}$ is the self dual Weyl spinor. The Weyl tensor $C_{a b c d}$ of $g_{a b}$ is given by

$$
\begin{aligned}
& C_{a b c d}=\sigma_{a}{ }^{A A^{\prime}}{\sigma_{b}}^{B B^{\prime}}{\sigma_{c}}^{C C^{\prime}} \sigma_{d}{ }^{D D^{\prime}} \\
& \left(\Psi_{A B C D} \varepsilon_{A^{\prime} B^{\prime}} \varepsilon_{C^{\prime} D^{\prime}}+\widetilde{\Psi}_{A^{\prime} B^{\prime} C^{\prime} D^{\prime} \varepsilon_{A B}{ }^{e} C D}\right),
\end{aligned}
$$

so that, if $\Psi_{A B C D}$ vanishes, $g_{a b}$ is self-dual and if $\widetilde{\Psi}_{A^{\prime} B^{\prime} C^{\prime} D^{\prime}}$ vanishes, it is anti-self dual. 
Let $\Sigma$ be a 3-dimensional submanifold of $M$. The unit normal, $\zeta^{a}$, to $\Sigma$ enables one to introduce the following additional structure. First, we have an isomorphism from the space of primed spinors on $\Sigma$ to the space of unprimed spinors:

$$
\lambda_{A^{\prime}} \rightarrow \tilde{\lambda}_{A}:=\sqrt{2} \lambda_{A^{\prime}} \zeta^{A^{\prime}}{ }_{A},
$$

where $\zeta^{A^{\prime} A}:=\sigma_{a}{ }^{A A^{\prime}} \zeta^{a}$. Second, we obtain an $S L(2, C)$ soldering form, $\sigma^{a}{ }_{A}{ }^{B}$ which maps second rank, trace-free unprimed spinors, $\lambda_{A}{ }^{B}$, to complex vectors tangent to $\Sigma: \lambda^{a}:=-\sigma^{a}{ }_{A}{ }^{B} \lambda_{B}{ }^{A} \equiv-\operatorname{Tr} \sigma^{a} \lambda$ via

$$
\sigma_{A B}^{a}=\sqrt{2} \zeta_{(A}{ }^{A^{\prime}} \sigma_{B) A^{\prime}}^{a},
$$

or

$$
\sigma_{A A^{\prime}}^{a}=\zeta^{a} \zeta_{A^{\prime} A}+\sqrt{2} \sigma_{A}^{a}{ }^{B} \zeta_{B A^{\prime}}
$$

Note that the spatial object $\sigma_{A B}^{a}$ is distinguised from the four dimensional $\sigma_{A A^{\prime}}$ by the latter having a primed and an unprimed spinor index, while the former has two unprimed spinor indices. Consequently, at points of $\Sigma$, unprimed spinors can be regarded either as entities associated with the 4-manifold $M$ or with the 3-manifold $\Sigma ; \lambda^{A} \bar{\mu}^{A^{\prime}} \sigma^{a}{ }_{A A^{\prime}}$ is a (complex) 4-vector, while $\lambda^{A} \tilde{\mu}^{B} \sigma^{a}{ }_{A B}$ is a (complex) 3-vector tangential to $\Sigma .^{2}$ Finally, the restriction of the connection ${ }^{4} \mathscr{D}$ to $\Sigma$ defines two 3-dimensional connections ${ }^{ \pm} \mathscr{D}$ on unprimed spinors and vectors:

$$
\begin{gathered}
{ }^{+} \mathscr{D}_{a} \lambda_{b B}:=q_{a}{ }^{m} q_{b}{ }^{n}{ }^{4} \mathscr{D}_{m} \lambda_{n B}, \\
-\mathscr{D}_{a} \tilde{\lambda}_{b B}:=\sqrt{2} q_{a}{ }^{m} q_{b}{ }^{n}\left({ }^{4} \mathscr{D}_{m} \tilde{\lambda}_{n B^{\prime}}\right) \zeta^{B^{\prime}}{ }_{B} .
\end{gathered}
$$

These are the Sen connections [4]. Using (14) and the definition of ${ }^{4} \mathscr{D}$ it follows that

$$
D_{a} \hat{\lambda}_{b B}:={ }^{ \pm} \mathscr{D}_{a} \lambda_{b B} \mp \sqrt{\frac{1}{2}} K_{a c} \sigma_{B}^{c}{ }^{A} \lambda_{b A},
$$

[where $K_{a b}$ is the extrinsic curvature (4) of $\Sigma$ ], is the unique torsion-free connection on $\Sigma$ which annihilates $\sigma_{A B}^{a}$, and $\varepsilon_{A B}$. While $D$ is determined by the intrinsic soldering form $\sigma_{A B}^{a}$, on $\Sigma$, one needs both the intrinsic soldering form and the extrinsic curvature of $\Sigma$ to define ${ }^{ \pm} \mathscr{D}$.

The curvature 2 -form, ${ }^{ \pm} F_{a b A}{ }^{B}$, of ${ }^{ \pm} \mathscr{D}$ is defined by:

$$
2^{ \pm} \mathscr{D}_{[a}{ }^{ \pm} \mathscr{D}_{b]} \lambda_{A}=:{ }^{ \pm} F_{a b A}{ }^{B} \lambda_{B} .
$$

Using (10), (11), and (15), we can relate it to the curvature of ${ }^{4} \mathscr{D}$. We have:

$$
{ }^{+} F_{a b A}{ }^{B}=q_{a}{ }^{m} q_{b}{ }^{n 4} F_{m n A}{ }^{B},
$$

and

$$
{ }^{-} F_{a b A}{ }^{B}=2 q_{a}{ }^{m} q_{b}{ }^{n}{ }^{4} F_{m n A^{\prime}}{ }^{B^{\prime}} \zeta_{A^{A}}{ }^{A^{\prime}} \zeta_{B^{\prime}}{ }^{B} .
$$

Recall that ${ }^{4} F_{m n A}{ }^{B}$ is an anti-self dual 2-form (and ${ }^{4} F_{m n A^{\prime}}{ }^{B^{\prime}}$ is a self dual 2-form.) Hence, ${ }^{+} F_{a b A}{ }^{B}$ vanishes if and only if ${ }^{4} F_{a b A}{ }^{B}$ vanishes, i.e. if and only if the Weyl

\footnotetext{
${ }^{2}$ Thus, the $S L(2, C)$ which acts on spinorial indices of $\sigma_{A B}^{a}$ is the complexification of the doublecovering of the 3-dimensional rotation group in the tangent space of $\Sigma$; it is not the doublecovering of the Lorentz group that acts in the tangent space of ${ }^{4} M$
} 
curvature of $g_{a b}$ is self-dual on $\sum$ (and ${ }^{-} F_{a b A}{ }^{B}$ vanishes if and only if it is anti-self dual). Thus the Sen connections, ${ }^{ \pm} \mathscr{D}$, are potentials for the (anti-) self dual part of the Weyl curvature on $\Sigma$.

Finally, if $g_{a b}$ is a real, positive definite metric, without loss of generality, one can impose certain Hermiticity restructions on $\sigma_{a}{ }^{A A^{\prime}}$ which are preserved only by an $S U(2)_{R} \times S U(2)_{L}$ subgroup of $S L(2, C) \times S L(2, C)$. Then, the primed spinors transform under $S U(2)_{R}$ [or self-dual part of the universal covering of $\left.S O(4)\right]$ and unprimed, under $S U(2)_{L}$ [or, the anti-self dual part of $\left.S O(4)\right]$. Given a 3-surface $\Sigma$, on the other hand, we also have "spatial spinors" transforming under the $S U(2)$ which is the double covering of the rotation group intrinsic to $\left(\Sigma, q_{a b}\right) .{ }^{4} F_{a b M}{ }^{N}$ and ${ }^{4} \widetilde{F}_{a b M}{ }^{N^{\prime}}$ are 2 -forms that take values in the Lie algebras of $S U(2)_{R}$ and $S U(2)_{L}$ respectively, while ${ }^{ \pm} F_{a b M}{ }^{N}$ takes values in the Lie algebra of spatial $S U(2)$.

Remark. Note that, a metric $g_{a b}$ defines an alternating tensor $\varepsilon_{a b c d}$ up to sign via

$$
\varepsilon_{a b c d} \varepsilon_{m n r s} g^{a m} g^{b n} g^{c r} g^{d s}=4 !
$$

In spite of the sign ambiguity, one can meaningfully ask if a given metric is half-flat, i.e., if its Riemann tensor satisfies

$$
{ }^{4} R_{a b c d}= \pm \frac{1}{2}{ }^{4} R_{m n c d} \varepsilon^{m n}{ }_{a b} .
$$

If we restrict ourselves to real Euclidean metrics, we can further divide half-flat metrics into two categories: self dual and anti-self dual. To do this, fix, once and for

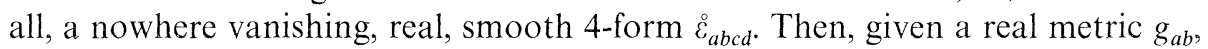
choose as its unique alternating tensor, the 4-form $\varepsilon_{a b c d}$ defined by (18) which has the same orientation as $\AA_{a b c d}$, i.e., which satisfies $\varepsilon_{a b c d}=f \AA_{a b c d}$, where $f$ is positive. Then, place $g_{a b}$ in the self dual category if its Riemann tensor satisfies (19) with the lower sign and in the anti-self dual category if it satisfies (19) with the upper sign. The labels "self-dual" and "anti-self dual" are convention dependent. However, the fact that an unambiguous classification exists is not. For a complex metric, on the other hand, this procedure fails because the function $f$ relating the metric compatible alternating tensor and the fiducial $\dot{\varepsilon}_{a b c d}$ is, in general, complex and the requirement that $\varepsilon_{a b c d}$ have the same orientation as $\varepsilon_{a b c d}$ is meaningless. However, if we have access to spinors, we can resolve the sign ambiguity by using the soldering form - square root of the metric - to select a preferred alternating tensor:

$$
\varepsilon^{a b c d}:=-\frac{1}{2} \sigma_{A A^{\prime}}^{[a} \sigma_{B B^{\prime}}^{b} \sigma_{C C^{\prime}}^{c} \sigma_{D D^{\prime}}^{d]}{ }^{A B}{ }_{\varepsilon}{ }^{C D} \varepsilon_{\varepsilon}^{A^{\prime} D^{\prime}} \varepsilon^{B^{\prime} C^{\prime}} .
$$

This choice of $\varepsilon^{a b c d}$ can be then used to determine if the Riemann tensor of a half-flat $\sigma_{A A^{\prime}}^{a}$ is self dual or anti-self dual.

\section{The New Variables}

In Sect. 2.A, we reduced Einstein's vacuum equation to constraints $\left(5^{\prime}\right)$ and $\left(6^{\prime}\right)$ and evolution equations $\left(7^{\prime}\right)$ and $\left(8^{\prime}\right)$ on the first and second fundamental forms, $q_{a b}$ and $K_{a b}$, of the 3-manifold $\Sigma$. Although the use of $q_{a b}$ and $K_{a b}$ is natural in that they admit a direct geometrical interpretation, the resulting equations are analytically quite complicated; $\left(8^{\prime}\right)$, in particular, involves non-polynomial functions of $q_{a b}$. Furthermore, none of these equations simplify if we require that $g_{a b}$ be half-flat. 
This has been the primary reason why the simplicity of the half-flat Einstein system has remained opaque from the Hamiltonian standpoint. In this section, we shall introduce new variables which are adapted to the half-flat condition and recast $\left(5^{\prime}\right)-\left(8^{\prime}\right)$ in terms of these variables.

The discussion of Sect. 2.B suggests that the Sen connections ${ }^{ \pm} \mathscr{D}$ should be used as some of the new variables. Let us therefore proceed as follows. Fix a real, 3-manifold $\Sigma$ and consider the trivial $S L(2, C)$ vector bundle $B$ on it. Each fiber of $B$ thus consists of a 2-complex dimensional vector space, equipped with a symplectic 2-form, which is preserved by the $S L(2, C)$ action. Cross sections of $B$ will be represented by fields, $\lambda_{A}, \mu_{A}, \ldots$ on $\Sigma, \lambda_{A}\left(x_{0}\right)$ being an element of the 2-dimensional fiber over $x_{0}$. By considering associated vector bundles, we acquire fields with arbitrary number of "internal" indices, e.g., $\alpha_{A \ldots B}{ }_{C \ldots D}$. We shall denote the symplectic 2-form by $\varepsilon_{A B}$ and its inverse by $\varepsilon^{A B}$ :

$$
\varepsilon_{A B} \varepsilon^{A D}=\delta_{B}^{D}
$$

and use it to "raise and lower" internal indices:

$$
\lambda^{A}=\varepsilon^{A B} \lambda_{B} \quad \text { and } \quad \lambda_{M}=\lambda^{N} \varepsilon_{N M} .
$$

Let us now consider connections $\mathscr{D}$ on these $S L(2, C)$ fields which annihilate $\varepsilon_{A B}$; $\mathscr{D}_{a} \lambda_{A B}=0$. Fix a connection $\partial$, chosen to be flat for simplicity. Then, any other connection can be written in terms of an $S L(2, C)$ Lie-algebra valued 1 -form, $A_{a A}{ }^{B}$ :

$$
\mathscr{D}_{a} \lambda_{A}=: \partial_{a} \lambda_{A}+A_{a A}{ }^{B} \lambda_{B} .
$$

These connection 1-forms will constitute a part of our new variables. The other part is essentially the soldering form: it is given by an isomorphism $\tilde{\sigma}^{a}{ }_{A}{ }^{B}$ between the 3-dimensional vector space of complex vector densities of weight one, $\lambda^{a}$, at any point $x_{0}$ of $\sum$ and that of trace-free linear operators, $\lambda_{A}{ }^{B}$, on the fiber over $x_{0}$, expressed by $\tilde{\lambda}^{a}=-\tilde{\sigma}^{a}{ }_{A}{ }^{B} \lambda_{B}{ }^{A} \equiv-\operatorname{Tr} \tilde{\sigma}^{a} \lambda{ }^{3}$ Given a soldering form, the internal indices get soldered to the tangent space of $\Sigma$ and can be regarded as $\operatorname{SL}(2, C)$ spinor indices.

Given a (density weighted) soldering form $\tilde{\sigma}_{A}^{a}{ }^{B}$, we obtain a complex metric $q_{a b}$ on $\sum$ as follows: $q_{a b}$ is the inverse of the non-degenerate, symmetric tensor field $q^{a b}$ defined by

$$
\operatorname{Tr} \tilde{\sigma}^{a} \tilde{\sigma}^{b}=-(\operatorname{det} q) q^{a b},
$$

where the density of weight two, $(\operatorname{det} q)$, is the determinant of $q_{a b}$. (Alternatively, we have $q^{a b}=\left(-\operatorname{det} \operatorname{Tr} \tilde{\sigma}^{a} \tilde{\sigma}^{b}\right)^{-1 / 2}\left(-\operatorname{Tr} \tilde{\sigma}^{a} \tilde{\sigma}^{b}\right)$.) Next, recall that a soldering form $\tilde{\sigma}^{a}$ defines a unique torsion-free connection $D$ on spinor and vector fields on $\Sigma$ via $D_{a} \tilde{\sigma}_{A}^{b}{ }^{B}=0$ (and $D_{a} \varepsilon_{A B}=0$ ). Hence, given a pair, $\left(\tilde{\sigma}^{a},{ }^{+} \mathscr{D}\right)$, where ${ }^{+} \mathscr{D}$ is an $\operatorname{SL}(2, C)$ connection on $\Sigma$, we acquire a field $\pi_{a A}{ }^{B}$ via:

$$
\begin{gathered}
\left({ }^{+} \mathscr{D}_{a}-D_{a}\right) \lambda_{A}=-\frac{1}{\sqrt{2}} \pi_{a A}{ }^{B} \lambda_{B}, \quad \text { or } \\
{ }^{+} A_{a A}{ }^{B}-\Gamma_{a A}{ }^{B}=\frac{1}{\sqrt{2}} \pi_{a A}{ }^{B},
\end{gathered}
$$

\footnotetext{
${ }^{3}$ Our notation is as follows: A tilde over a letter implies that the letter represents a density of weight one while a tilde under a letter implies that it represents a density of weight minus one
} 
where $\Gamma_{a A}{ }^{B}$ is the (spin-) connection 1-form of $D$. Note, however, that unlike $K_{a b}$ of (16),

$$
\pi_{a b}:=-(\operatorname{det} q)^{-1 / 2} \operatorname{Tr}\left(\pi_{a} \sigma^{d}\right) q_{b d}
$$

is not necessarily symmetric in $a$ and $b$. Let us define $K_{a b}$ via

$$
K_{a b}=\pi_{(a b)} .
$$

Then, given any pair, $\left(\tilde{\sigma}^{a},{ }^{+} \mathscr{D}_{a}\right)$, for $\left(\tilde{\sigma}^{a},{ }^{+} A_{a}\right)$, we acquire a pair $\left(q_{a b}, K_{a b}\right)$ consisting of a (complex) metric $q_{a b}$ and a symmetric tensor field $K_{a b}$, which can be used as first and second fundamental forms, i.e., "initial data", for Eqs. $\left(5^{\prime}\right)-\left(8^{\prime}\right)$ of Sect. 2.A. We shall now regard $\left(\tilde{\sigma}^{a},{ }^{+} A_{a}\right)$ as the primary objects and $\left(q_{a b}, K_{a b}\right)$ as secondary, derived quantities.

Note that, given a pair, $\left(\tilde{\sigma}^{a},{ }^{-} A_{a}\right)$, where ${ }^{-} A_{a}$ is any $\operatorname{SL}(2, C)$-valued connection 1-form, we can define a pair $\left(q_{a b}, K_{a b}\right)$ in the following alternate way: $q_{a b}$ is defined as above but $K_{a b}$ is defined via:

$$
\begin{gathered}
-A_{a A}{ }^{B}-\Gamma_{a A}{ }^{B}=-\frac{1}{\sqrt{2}} \pi_{a A}{ }^{B}, \\
K_{a b}=\pi_{(a b) .}
\end{gathered}
$$

[This alternate definition is motivated by (16) with lower signs.] As geometric objects in their own right, there is no distinction between ${ }^{+} A_{a}$ and ${ }^{-} A_{a}$; they are both $S L(2, C)$-valued connection 1 -forms. However, the rules that yield us initial data $\left(q_{a b}, K_{a b}\right)$ starting from $\left(\tilde{\sigma}^{a},{ }^{+} A_{a}\right)$ and $\left(\tilde{\sigma}^{a},{ }^{-} A_{a}\right)$ are different. Thus, \pm signs refer to the two rules, $\left(16^{\prime}\right)$ and $\left(16^{\prime \prime}\right)$. Since, for our present purpose, we are interested more in the relation between $S L(2, C)$-connections and geometry of space-time than in the connections in their own right, we shall always associate a prefix + or - to the connection 1-forms, thereby emphasizing the specific way in which we wish to use them. In what follows, we can work either with $\left(\tilde{\sigma}^{a},{ }^{+} A_{a}\right)$ or $\left(\tilde{\sigma}^{a},{ }^{-} A_{a}\right)$ and \pm will always stand for + or - .

Let us now rewrite Eqs. $\left(5^{\prime}\right)-\left(8^{\prime}\right)$ in terms of new variables. The motivation behind the procedure that follows comes from a Hamiltonian framework [6]. Here, we shall present only the final picture.

Let us begin with constraints. In the passage to new variables, we have introduced three new degrees of freedom: while $q_{a b}$ has six components per space point, $\tilde{\sigma}^{a}{ }_{A}{ }^{B}$ has nine. Hence the set of equations in terms of new variables contain three new constraints. These turn out to be

$$
{ }^{ \pm} \mathscr{D}_{a} \tilde{\sigma}^{a}=\partial_{a} \tilde{\sigma}^{a}+\left[{ }^{ \pm} A_{a}, \tilde{\sigma}^{a}\right]=0,
$$

which, using $\left(16^{\prime}\right),\left(16^{\prime \prime}\right),(22)$ and the fact that $D$ annihilates $\tilde{\sigma}^{a}$, reduce to:

$$
\pi_{[a b]}=0 \text { or } \pi_{a b}=K_{a b} .
$$

[Note that, Christoffel symbols do not appear in (23) because we are computing the divergence of a vector density.] The remaining constraints are the same as before, Eqs. $\left(5^{\prime}\right)$ and $\left(6^{\prime}\right)$. To express them in terms of $\tilde{\sigma}^{a}$ and ${ }^{ \pm} A_{a}$, let us first compute the curvature of ${ }^{ \pm} A_{a}$. Using $\left(16^{\prime}\right)$ and $\left(16^{\prime \prime}\right)$ one finds thats ${ }^{ \pm} F_{a b A}{ }^{B} \lambda_{B}$ $:=2^{ \pm} \mathscr{D}_{[a}{ }^{ \pm} \mathscr{D}_{b]} \lambda_{A}$ is given by

$$
{ }^{ \pm} F_{a b A}{ }^{B}=R_{a b A}{ }^{B}-\frac{1}{\sqrt{2}} \varepsilon_{c d e} \pi_{a}{ }^{d} \pi_{b}{ }^{e} \sigma_{A}^{c}{ }^{B} \pm \sqrt{2} D_{[a} \pi_{b] A}{ }^{B},
$$


where $R_{a b A}{ }^{B}$ is the spinorial curvature of $D, \sigma^{a}=(\operatorname{det} q)^{-1 / 2} \tilde{\sigma}^{a}$ and $\varepsilon_{a b c}$ is the metric compatible 3-form defined by

$$
-\sqrt{2}\left(\operatorname{Tr} \sigma^{a} \sigma^{b} \sigma^{c}\right) \varepsilon_{a b c}=3 !
$$

Contracting (24) with $\sigma_{B}^{a}{ }^{A}$, one obtains

$$
\begin{aligned}
\operatorname{Tr} \sigma^{a \pm} F_{a b} & =\frac{1}{\sqrt{2}} \pi_{a m} \pi_{b n} \varepsilon^{m n a} \mp \frac{1}{\sqrt{2}} D^{a}\left(\pi_{b a}-\pi q_{b a}\right) \\
& \simeq \mp \frac{1}{\sqrt{2}} D^{a}\left(K_{a b}-K q_{a b}\right),
\end{aligned}
$$

where $\simeq$ stands for equality modulo the new constraint (23). Similarly, we have

$$
\begin{aligned}
\operatorname{Tr} \sigma^{a} \sigma^{b \pm} F_{a b} & =\frac{1}{2}\left(R+\pi^{2}-\pi_{a b} \pi^{b a}\right) \mp \varepsilon^{a b c} D_{a} \pi_{b c} \\
& \simeq \frac{1}{2}\left(R+K^{2}-K_{a b} K^{a b}\right) .
\end{aligned}
$$

Consequently, modulo (23), (5') and (6') can be respectively written as:

$$
\begin{gathered}
\operatorname{Tr} \tilde{\sigma}^{a \pm} F_{a b}=0, \quad \text { and } \\
\operatorname{Tr} \tilde{\sigma}^{a} \tilde{\sigma}^{b \pm} F_{a b}=0 .
\end{gathered}
$$

Thus, we have re-expressed the constraints in terms of new variables $\tilde{\sigma}^{a}$ and ${ }^{ \pm} A_{a}$ : The satisfaction of $(23),\left(5^{\prime \prime}\right)$ and $\left(6^{\prime \prime}\right)$ by $\left(\tilde{\sigma}^{a},{ }^{ \pm} A_{a}\right)$ implies that the pair $\left(q_{a b}, K_{a b}\right)$ determined from $\left(\tilde{\sigma}^{a},{ }^{ \pm} A_{a}\right)$ via (21) and (22) satisfies the Einstein constraints $\left(5^{\prime}\right)$ and $\left(6^{\prime}\right)$. Equations (23), $\left(5^{\prime \prime}\right)$ and $\left(6^{\prime \prime}\right)$ are, furthermore, simpler because they are polynomial in terms of the new variables.

In the Hamiltonian formulation of general relativity, there is a close interrelation between the Hamiltonian generating time-translation and the scalar constraint $\left(6^{\prime}\right)$ [or $\left.\left(6^{\prime \prime}\right)\right]$. Using the relation, one can obtain evolution equations for $\tilde{\sigma}^{a}$ and ${ }^{ \pm} A_{a}[6]$. These are:

$$
\begin{gathered}
\dot{\sigma}^{a}= \pm 2 \sqrt{2} \pm \mathscr{D}_{b}\left(\mathrm{~N}_{\sim} \tilde{\sigma}^{[a} \tilde{\sigma}^{b]}\right), \text { and } \\
\pm \dot{A}_{a}= \pm \sqrt{2} \underset{\sim}{\mathrm{N}}\left[\tilde{\sigma}^{b},{ }^{ \pm} F_{a b}\right],
\end{gathered}
$$

where the lapse $\mathrm{N}$ is now a scalar density of weight minus one, related to the scalar lapse $N$ of Sect. 2 .A via $N=(\operatorname{det} q)^{1 / 2} N$. Again, using the expressions (21) and (22) of $q_{a b}$ and $K_{a b}$ in terms of $\left(\tilde{\sigma}^{a},{ }^{ \pm} A_{a}\right)$, it follows that $\left(7^{\prime \prime}\right)$ and $\left(8^{\prime \prime}\right)$ imply $\left(7^{\prime}\right)$ and $\left(8^{\prime}\right)$.

To summarize, given a 4-manifold $M$, topologically $\Sigma \times R$, and a pair of fields $\left(\tilde{\sigma}^{a}(t),{ }^{ \pm} A_{a}(t)\right)$ defined on each leaf $t=$ const of the foliation, satisfying $(23),\left(5^{\prime \prime}\right)-\left(8^{\prime \prime}\right)$, we obtain a pair of fields $\left(q_{a b}(t), K_{a b}(t)\right)$ on $M$ satisfying $\left(5^{\prime}\right)-\left(8^{\prime}\right)$, and hence a 4 metric $g_{a b}$ satisfying the vacuum Einstein equation. Using (3), it follows that the 4metric can be written as

$$
g^{a b}=q^{a b}+\zeta^{a} \zeta^{b}=(\operatorname{det} q)^{-1}\left(-\operatorname{Tr} \tilde{\sigma}^{a} \tilde{\sigma}^{b}+(\mathbf{N})^{-2} t^{a} t^{b}\right),
$$

where, as in Sect. 2.A, $t^{a}$ is a vector field on $M$ defined by $t^{a}:=\left(\mathscr{L}_{\zeta} t\right)^{-1} \zeta^{a} \equiv N \zeta^{a}$. We shall see in the next section that the form (23), $\left(5^{\prime \prime}\right)-\left(8^{\prime \prime}\right)$ of Einstein's equation is especially well-suited to analyze half-flat metrics. 
Remarks. i) Mathematically, one may regard a pair $\left(\tilde{\sigma}^{a},{ }^{ \pm} A_{a}\right)$ as representing an "initial datum" for $S L(2, C)$ Yang-Mills theory, ${ }^{ \pm} A_{a}$ playing the role of the YangMills connection and $\tilde{\sigma}^{a}$, of the Yang-Mills electric field. Then,(23) is just the Gauss law constraint for Yang-Mills theory. To qualify as an Einstein "initial datum," on the other hand, $\left(\tilde{\sigma}^{a},{ }^{ \pm} A_{a}\right)$ has to satisfy four additional constraints $\left(5^{\prime \prime}\right),\left(6^{\prime \prime}\right)$. Thus, we have an imbedding of the space of Einstein initial data into that of the YangMills initial data.

ii) The reduction to the Euclidean case is obtained simply by restricting $\tilde{\sigma}^{a}$, and ${ }^{ \pm} A_{a}$ to be Hermitian with respect to some Hermitian conjugation operation. Then, $q^{a b}$, defined by (21), is guaranteed to be a positive definite 3 -metric on $\Sigma$ and $K_{a b}$ of (22), a real second rank tensor field.

iii) It is only for simplicity that we have restricted the fiducial connection $\hat{\partial}$ to be flat. It is straightforward to extend the framework to the case when it is not: The form of the constraint and evolution equations remains the same; only the expression of ${ }^{ \pm} F_{a b}$ in terms of ${ }^{ \pm} A_{a}$ acquires additional terms. Such an extension is essential when the topology of $\Sigma$ is non-trivial.

i) In the general, complex case, $(\operatorname{det} q)^{1 / 2}$ is unique only up to sign. Hence, given $\left(\tilde{\sigma}^{a},{ }^{ \pm} A_{a}\right)$, we can recover $\sigma^{a}$ and $K_{a b}$ only up to sign. These ambiguities do not affect the fact that, modulo (23), $\left(5^{\prime \prime}\right)$ is equivalent to $\left(5^{\prime}\right)$ and $\left(6^{\prime \prime}\right)$ to $\left(6^{\prime}\right)$. However, the situation with evolution equations is more complicated: if we change the sign of $(\operatorname{det} q)^{1 / 2}$ - and hence of $\sigma^{a}$ and $K_{a b}$ - we have to simultaneously change the sign of the "dot operation" (i.e. the sign of $\left.\zeta^{a}\right)$ in $\left(7^{\prime \prime}\right)$ and $\left(8^{\prime \prime}\right)$. The 3 -metric $q^{a b}$ as well as the 4-metric $g_{a b}$ are, however, unaffected. In the real, Riemannian case, one can avoid all sign ambiguities simply by requiring that $(\operatorname{det} q)^{1 / 2}$ be positive.

v) A primary limitation of this framework is that the topology of $M$ is required to be of the type $\Sigma \times R$. Many Euclidean instantons, for example, do not accomodate such a topology. On the other hand, the $3+1$ form of field equations in terms of $\left(\tilde{\sigma}^{a},{ }^{+} A_{a}\right)$ is such that one never needs to lower the $a$ vector index on $\tilde{\sigma}^{a}$. Consequently, Eqs. (23), $\left(5^{\prime \prime}\right)-\left(8^{\prime \prime}\right)$ represent a generalization of Einstein's equation, reducing to it when $\tilde{\sigma}^{a}$ is non-degenerate. It is quite possible that solutions to $(23),\left(5^{\prime \prime}\right)-\left(8^{\prime \prime}\right)$ exist in which $\tilde{\sigma}^{a}$ fails to be invertible, say, on a set of measure zero of $M$. Then, the resulting solution $g_{a b}$ will not be invertible at such points and the domain on which $g_{a b}$ satisfies Einstein's equation in the traditional sense may have an interesting topology.

\section{The Half-Flat Case}

The discussion in Sect. 2.A implies that ${ }^{-} A_{a}$ is a potential for the self dual part of the Weyl tensor and ${ }^{+} A_{a}$, for the anti-self dual part. One can see this explicitly in the $3+1$ framework as follows. Define ${ }^{ \pm} F_{a b c}$ by ${ }^{ \pm} F_{a b}{ }^{c}=-\operatorname{Tr} \sigma^{c \pm} F_{a b}$. Then, by dualizing on $a$ and $b$ in (24), one obtains:

$$
{ }^{ \pm} W^{c d}:=\varepsilon^{a b d}{ }^{ \pm} F_{a b}{ }^{c} \simeq \sqrt{2}\left(R^{c d}+K^{c}{ }_{m} K^{m d}-K K^{c d} \pm \varepsilon^{a b c} D_{a} K_{b}{ }^{d}\right),
$$

where, $\simeq$ now stands for equality modulo constraints $(23),\left(5^{\prime \prime}\right)$, and $\left(6^{\prime \prime}\right)$. In a Ricciflat space-time, one can express the electric and the magnetic parts of the Weyl tensor, $E_{a b}:=C_{a m b n} \zeta^{m} \zeta^{n}$ and $B_{a b}:={ }^{*} C_{a m b n} \zeta^{m} \zeta^{n}$, in terms of the initial data 
$\left(q_{a b}, K_{a b}\right)$, and, using (28.a), show that:

$$
{ }^{ \pm} W^{c d}=\sqrt{2}\left(E^{c d} \pm B^{c d}\right) .
$$

Hence, if ${ }^{ \pm} A_{a}=0,{ }^{ \pm} W^{c d}$ vanishes, whence $E^{c d}=\mp B^{c d}$, i.e., the Weyl tensor is, respectively, self-dual or anti-self dual. This is why, the reformulation $(23),\left(5^{\prime \prime}\right)-\left(8^{\prime \prime}\right)$, of Einstein's equation in terms of $\left(\tilde{\sigma}^{a},{ }^{ \pm} A_{a}\right)$ is well adapted to the analysis of half-flat space-times.

To be specific, let us consider self dual space-times. Then, it is easier to work with $\left(\tilde{\sigma}^{a},{ }^{+} A_{a}\right)$ rather than $\left(\tilde{\sigma}^{a},{ }^{-} A_{a}\right)$. As we just saw, self duality means the vanishing of ${ }^{+} F_{a b}$. For simplicity, let us assume that we can go to a gauge in which ${ }^{+} A_{a}$ vanishes on $\Sigma$. Then, constraints $\left(5^{\prime \prime}\right)$ and $\left(6^{\prime \prime}\right)$ (with upper signs) are trivially satisfied and (23) reduces to:

$$
\partial_{a} \tilde{\sigma}^{a}=0 .
$$

Of the "evolution equations", $\left(8^{\prime \prime}\right)$ simply insures that if ${ }^{+} A_{a}$ vanishes initially, it remains zero. Finally, $\left(7^{\prime \prime}\right)$ simplifies to:

$$
\dot{\tilde{\sigma}}^{a}=2 \sqrt{2} \partial_{b}\left(\mathbf{N} \tilde{\sigma}^{[a} \tilde{\sigma}^{b]}\right)
$$

Thus, in the self dual case, Einstein's equation reduces to two first order equations on $\tilde{\sigma}^{a}$, a linear constraint equation $\left(1^{\prime}\right)$ and a non-linear "evolution equation" $\left(2^{\prime}\right)$. These equations can be further simplified by choosing the lapse $\underset{\sim}{N}$ suitably. Let $\underset{\sim}{\mathrm{N}}$ be time-independent and let it satisfy $\partial_{a} N=0$ on $\Sigma$. [Since $\partial_{a}$ is flat, we can just set $N=(\operatorname{det} q)^{-1 / 2}$, where $\stackrel{q}{a b}_{a b}$ is a flat metric annihilated by $\partial$.] Next, let us introduce a triad $V^{a},(\mathbf{i}=1,2,3)$ of vector fields on $\Sigma$ and expand out the (undensitized) soldering form $N_{\sim} \tilde{\sigma}^{a}{ }_{A}{ }^{B}$ in terms of (fixed, constant) Pauli matrices $\tau^{\mathbf{i}}{ }_{A}{ }^{B}$ and the triad $V^{a}$ :

$$
\mathrm{N} \tilde{\sigma}^{a}{ }_{A}{ }^{B}=V^{a}{ }_{\mathbf{i}} \tau^{\mathbf{i}}{ }_{A}{ }^{B}
$$

where $\tau^{\mathbf{i}}$ are so normalized as to satisfy

$$
\left[\tau^{\mathbf{i}}, \tau^{\mathbf{j}}\right]=\sqrt{2} \varepsilon^{\mathbf{i j k}} \tau^{\mathbf{k}} .
$$

Then, multiplying (1') by $\underset{\sim}{\mathrm{N}}$ and using (29), we have

$$
\partial_{a}\left(V^{a}{ }_{\mathbf{i}} \tau^{\mathbf{i}}{ }^{B}\right)=0
$$

or, using the constancy and the non-degeneracy of the Pauli matrices,

$$
\partial_{a} V^{a}{ }_{\mathbf{i}}=0 \text {. }
$$

Next, multiplying (2') by $\underset{\sim}{\mathrm{N}}$ and using (29) and (1), we have:

$$
\begin{aligned}
\dot{V}^{a}{ }_{\mathbf{i}} \tau^{i} & =\sqrt{2}\left(\partial_{b} V^{a}{ }_{\mathbf{i}} V^{b}{ }_{\mathbf{j}}\right)\left[\tau^{\mathbf{i}}, \tau^{\mathbf{j}}\right] \\
& =2 \varepsilon^{\mathbf{i j k}}\left(V^{b}{ }_{\mathbf{i}} \partial_{b} V^{a}{ }_{\mathbf{j}}\right) \tau^{\mathbf{k}} \\
& =\varepsilon^{\mathbf{i} \mathbf{j k}}\left[V_{\mathbf{i}}, V_{\mathbf{j}}\right]^{a} \tau^{\mathbf{k}},
\end{aligned}
$$

whence, non-degeneracy of $\tau^{\mathbf{i}}$ implies:

$$
\dot{V}_{\mathbf{k}}^{a}=\varepsilon_{\mathbf{i j k}}\left[V_{\mathbf{i}}, V_{\mathbf{j}}\right]^{a} .
$$


Thus, the self dual Einstein equation is reduced to Eqs. (1) and (2) on a field of triads on $\Sigma$. Finally, let us express the required half-flat four metric $g_{a b}$ in terms of a solution $V^{a}{ }_{\mathbf{i}}(t)$ to (1) and (2) on $\Sigma \times R$. We have, from (3'),

with,

$$
\begin{aligned}
g^{a b} & =(\operatorname{det} q)^{-1} \underset{\sim}{N^{-2}}\left(-\operatorname{Tr}\left(\underset{\sim}{\mathbf{N}} \tilde{\sigma}^{a}\right)\left(\mathrm{N}_{\sim} \tilde{\sigma}^{b}\right)+t^{a} t^{b}\right) \\
& =(\operatorname{det} \hat{q})^{1 / 2} \underset{\sim}{\mathrm{N}}\left(\hat{q}^{a b}+t^{a} t^{b}\right),
\end{aligned}
$$

$$
\hat{q}^{a b}=V^{a}{ }_{\mathbf{i}} V^{b} \delta^{\mathbf{i j}} .
$$

Here we have used the fact that the definitions of $q^{a b}, \hat{q}^{a b}$, and $\mathbf{N}$ imply the relation $(\operatorname{det} q)^{2}(\operatorname{det} \hat{q}) \mathbb{N}^{6}=1$.

Let us summarize and state the main result. Fix a 4-manifold $M=\Sigma \times R$, where $\Sigma$ is a 3-manifold. Let the leaves of the natural foliation of $M$ be labelled by constant values of a coordinate $t$ and let $t^{a}$ be the natural "vertical vector field" whose affine parameter is given by $t$. Fix a ( $t$-independent) flat connection $\partial$ on leaves $t=$ const of the foliation. Then, each solution $V^{a}{ }_{\mathbf{i}}(x, t), x \in \Sigma$, of

and

$$
\partial_{a} V^{a}{ }_{\mathbf{i}}=0 \text {, }
$$

$$
\mathscr{L}_{t} V^{a}{ }_{\mathbf{i}}=\varepsilon_{\mathbf{i j k}}\left[V_{\mathbf{j}}, V_{\mathbf{k}}\right]^{a}
$$

defines a self dual, Ricci-flat metric $g_{a b}$ via $\left(3^{\prime \prime}\right)$, where $\mathrm{N}$ is given, up to a multiplicative constant, by $\mathscr{L}_{t} \mathrm{~N}=0$ and $\partial_{a} \mathrm{~N}=0$. Conversely, given a Ricci-flat, self dual metric $g_{a b}$ on $M$, the field ${ }^{+} F_{a b A}{ }^{B}$ it induces on each leaf of the foliation vanishes, whence the spatial soldering forms $\tilde{\sigma}^{a}{ }_{A}{ }^{B}$ satisfy (23) and (7"). If $\Sigma$ admits a flat spin-connection, one can introduce a field of triads $V^{a}{ }_{\mathbf{i}}(x, t)$ on $M$, satisfying (1) and (2). Finally, the metric $g_{a b}$ is real and positive definite if and only if the initial field of triads, $V_{i}^{a}(x, 0)$ is real.

Remarks. i) To obtain anti-self dual solutions, it is simplest to work with $\left(\tilde{\sigma}^{a},{ }^{-} A_{a}\right)$ and use Eqs. (23), $\left(5^{\prime \prime}\right)-\left(8^{\prime \prime}\right)$ with lower (minus) sign. Anti-self duality implies ${ }^{-} F_{a b}=0$. Then, $\left(5^{\prime \prime}\right)$ and $\left(6^{\prime \prime}\right)$ are identically satisfied. By choosing the gauge in which - $A_{a}$ vanishes, $\left(8^{\prime \prime}\right)$ becomes redundant and (23) and ( $\left.7^{\prime \prime}\right)$ reduce to, respectively,

$$
\partial_{a} \tilde{\sigma}^{a}=0 \quad \text { and } \quad \dot{\tilde{\sigma}}^{a}=-2 \sqrt{2} \partial_{b}\left(N_{\sim} \tilde{\sigma}^{[a} \tilde{\sigma}^{b]}\right) .
$$

By choosing the lapse and triads as before, these reduce to

$$
\partial_{a} V^{a}{ }_{\mathbf{i}}=0 \text { and } \dot{V}^{a}{ }_{\mathbf{i}}=-\varepsilon_{\mathbf{i j k}}\left[V_{\mathbf{j}}, V_{\mathbf{k}}\right]^{a} .
$$

One could, of course, work with $\left(\tilde{\sigma}^{a},{ }^{+} A_{a}\right)$ even in the anti-self dual case, although, in this case, the equations are substantially more complicated. A solution to the anti-self dual equations directly in terms of $\left(\tilde{\sigma}^{a},{ }^{+} A_{a}\right)$ would correspond to the solution of the so-called "googly problem" in the twistor program [8], which asks one to solve the anti-self dual equation directly on the dual twistor space, rather than the twistor space.

ii) Recall that, in the general complex case, starting from $\left(\tilde{\sigma}^{a},{ }^{+} A_{a}\right)$, one can recover $(\operatorname{det} q)^{-1 / 2}, \sigma^{a}$, and $K_{a b}$ only up to sign. Given a $\sigma^{a}$, however, one can select a preferred 3-orientation, $\varepsilon_{a b c}$, by fixing a convention:

$$
\varepsilon^{a b c}:=-\sqrt{2} \operatorname{Tr} \sigma^{a} \sigma^{b} \sigma^{c} .
$$


Thus, once we fix $(\operatorname{det} q)^{1 / 2}$ by choosing one sign over another, $\sigma^{a}, K_{a b}$, and $\varepsilon^{a b c}$ are all uniquely determined and they all change sign if we change the sign of $(\operatorname{det} q)^{1 / 2}$. Consequently, Eq. (28) and the subsequent 3-dimensional formulation of the distinction between self dual and anti-self dual configurations is unaffected by sign ambiguities.

iii) Given a pair $\left(\tilde{\sigma}^{a},{ }^{+} A_{a}\right)$, the 4-dimensional orientation can be fixed ambiguously. Fix a convention for $(\operatorname{det} q)^{1 / 2}$. Then $q_{a b}, K_{a b}$, and $\varepsilon^{a b c}$ are unique. One can now select $\zeta^{a}$ uniquely by requiring $\mathscr{L}_{\zeta} q_{a b}=K_{a b}$ and then set

$$
\varepsilon^{a b c d}=4 \varepsilon^{[a b c \zeta d]} \text {. }
$$

If one changes the convention on $(\operatorname{det} q)^{1 / 2}, K_{a b}, \zeta^{a}$, and $\varepsilon^{a b c}$ all change sign leaving $\varepsilon^{a b c d}$ unaffected. This choice of $\varepsilon^{a b c d}$ - which is equivalent to that made through $\left(18^{\prime}\right)$ - underlies our classification of half-flat metrics into self dual and anti-self dual categories.

iv) Note that, given a solution $\left(\tilde{\sigma}^{a},{ }^{+} A_{a}=0\right)$ to the self dual equations $\left(1^{\prime}\right)$ and $\left(2^{\prime}\right),\left(-\tilde{\sigma}^{a},{ }^{-} A_{a}=0\right)$ solves the anti-self dual equations given above. It is easy to verify that, while the 4-metric $g_{a b}$ is the same in the two cases, the orientation $\varepsilon^{a b c d}$ is of opposite sign. Thus, we see once again that the 4-metric can be only called halfflat. In the real, Riemannian case, one can do better. One can fix a fiducial $\varepsilon^{\text {aabcd }}$ and admit only those $\left(\tilde{\sigma}^{a},{ }^{ \pm} A_{a}\right)$ which yield an $\varepsilon^{a b c d}$ with the same orientation as $\varepsilon^{\text {abcd }}$. Then, if $\left(\tilde{\sigma}^{a},{ }^{+} A_{a}=0\right)$ is admissible, $\left(-\tilde{\sigma}^{a},{ }^{-} A_{a}=0\right)$ is not, whence a given half-flat metric $g_{a b}$ is either self dual or anti-self dual and the redundancy present in the general complex case is avoided.

\section{Structure of Equations (1) and (2)}

This section is divided into two parts. In the first, we use Eqs. (1) and (2) to obtain, almost trivially, a class of half-flat metrics and in the second we analyze the linearized version of these equations.

\section{A Plane-Fronted Solutions}

Let $\Sigma$ be topologically $R^{3}$. Introduce on $\Sigma$, a global chart, $(x, y, z)$, and make the following ansatz for the triad $V^{a}$ :

$$
\begin{aligned}
& V_{1}=a(z, t) \partial_{x}+b(z, t) \partial_{y}, \\
& V_{2}=c(z, t) \partial_{x}+d(z, t) \partial_{y}, \\
& V_{3}=\partial_{z} .
\end{aligned}
$$

Let $\partial$ be the flat (time independent) connection on $\Sigma$ given by the chart. Then the triad (30) automatically satisfies the constraint equation (1) and the evolution equation (2) for $V_{3}$ on $\sum \times R$. The remaining evolution equations impose restrictions on functions $a, b, c, d$ :

$$
\dot{V}_{1}=-V_{2}^{\prime}, \quad \dot{V}_{2}=V_{1}^{\prime},
$$

where prime denotes differentiation with respect to the $z$-coordinate. The general solution to these equations is given by:

$$
V_{1}=(V+\tilde{V}) / 2, \quad V_{2}=(V-\tilde{V}) / 2 i,
$$


where

$$
V=\alpha \partial_{x}+\beta \partial_{y}, \quad \tilde{V}=\tilde{\alpha} \hat{\partial}_{x}+\widetilde{\beta} \partial_{y} .
$$

Here $\alpha, \beta$ and $\tilde{\alpha}, \tilde{\beta}$ are arbitrary holomorphic functions of $z+i$ and $z$-it, respectively. Thus, we have obtained a large class - worth 4 holomorphic functions of one complex variable - of solutions to (1) and (2) by a trivial integration.

Using $\left(3^{\prime \prime}\right)$, one can now construct the inverse 4-metric $g^{a b}$. Evaluating its components in the $x, y, z, t$ chart and taking the inverse of the resulting $4 \times 4$ matrix, one obtains:

$$
g_{a b} d x^{a} d x^{b}=D^{-1}\left[(\beta \widetilde{\beta}) d x^{2}-(\alpha \widetilde{\beta}+\tilde{\alpha} \beta) d x d y+\alpha \tilde{\alpha} d y^{2}\right]+D\left[d z^{2}+d t^{2}\right],
$$

where $D=(\alpha \tilde{\beta}-\tilde{\alpha} \beta) / 2 i$. Each of these metrics is a (half-flat) solution of the vacuum Einstein equation. Since the metric coefficients depend only on $z$ and $t$, these metrics have two killing fields, $\partial_{x}$ and $\partial_{y}$. Next, since the dependence of the coefficients on $z$ and $t$ is via $z \pm i t$, by analogy with linearized Einstein's theory, the solutions may be called plane-fronted metrics. Finally, if we set $\tilde{\alpha}=\bar{\alpha}$ and $\widetilde{\beta}=\bar{\beta}$, the resulting metrics are all real and positive definite.

Since the primary purpose of this subsection is only to show, by illustration, that Eqs. (1) and (2) provide a powerful tool to obtain half-flat 4-metrics, we shall not analyse detailed properties of metrics (33). We only note that it is not difficult to introduce other ansätze to trivially integrate (1) and (2), and hence the self dual Einstein equation. For example, Ashtekar and Mazur have found all (Bianchi type) solutions in which the triad $V^{a}{ }_{\mathbf{i}}$ has the commutation relations of any threedimensional Lie algebra.

\section{B Linearization}

In order to reconcile the main result of this paper with our physical intuition about half-flat solutions, let us now linearize Eqs. (1) and (2) off flat space and see if the physical degrees of freedom in the resulting solutions correspond precisely to right circularly polarized weak gravitational waves.

Let $M=R^{4}$. Fix a flat Riemannian metric $\eta_{a b}$ on $M$. Foliate $M$ by 3-planes and introduce a covariantly constant orthonormal tetrad $\left(t^{a}, V^{a}\right)$ adapted to the foliation. $V^{a}{ }_{i}$ will be our background configuration. To linearize, we will want to consider a one parameter family of metrics $g_{a b}(s)$ on $M$ such that $g_{a b}(0)=\eta_{a b}$.

Since the right circularly polarized metrics are usually written in a gauge in which they are transverse, traceless and orthogonal to $t^{a}$ and since

$$
g^{a b}(s)=(\operatorname{det} \hat{q}(s))^{1 / 2} \underset{\sim}{N}(s)\left(\hat{q}^{a b}(s)+t^{a}(s) t^{b}(s)\right),
$$

it is convenient to work in a gauge in which $N(s)(\operatorname{det} \hat{q}(s))^{1 / 2}=1$ and $t^{a}(s)=t^{a}$. Thus we are led to consider a 1-parameter family, $V^{a}{ }_{\mathbf{i}}(s)$, of solutions to:

and

$$
(\operatorname{Div}(s)) V^{a}(s)=0 \text {, }
$$

$$
\dot{V}_{\mathbf{i}}^{a}(s)=\varepsilon_{\mathbf{i} \mathbf{j k}}\left[V_{\mathbf{j}}(s), V_{\mathbf{k}}(s)\right]^{a}
$$

on $M$, with $V^{a}{ }_{i}(0)=V^{a}$, where dot denotes Lie derivative with respect to the fixed, $s$-independent vector field $t^{a}$ and $\operatorname{Div}(s)$ is defined with respect to the volume 
element $N(s)^{-1}=[\operatorname{det} \hat{q}(s)]^{1 / 2}$. The linearized field is:

$$
L_{\mathbf{i}}^{a}:=\left.\frac{d}{d s} V^{a}(s)\right|_{s=0},
$$

and it satisfies the linearized versions of (34) and (35):

$$
\partial_{a} L_{\mathbf{i}}^{a}-V^{b} \partial_{b}\left(L_{\mathbf{j}}^{a} V_{a}^{\mathbf{j}}\right)=0
$$

and

$$
\dot{L}_{\mathbf{i}}^{a}=2 \varepsilon_{\mathbf{i j k}}\left[V_{\mathbf{j}}, L_{\mathbf{k}}\right]^{a} .
$$

Set $L_{a b}=L_{a \mathbf{i}} V_{b}^{\mathbf{i}}$ and consider the time evolution of its antisymmetric part, $L_{[a b]}$. It follows immediately from (38) and (37) that $L_{[a b]}$ is time independent. Thus, as one might expect, only the symmetric part of $L_{a b}$ carries dynamical information. In fact, by taking the "dot" of (37) and using (38) one obtains, after some simplification: ${ }^{4}$

$$
0=\partial_{a} \dot{L}_{b}-\partial_{b} \dot{L}=-2 \partial^{a} \partial_{a}\left(\varepsilon_{b}^{c d} L_{c d}\right)
$$

whence, using boundary conditions on $L_{a b}$ we conclude

$$
L_{[a b]}=0 \text {. }
$$

Thus, in the gauge we have chosen thus far, linearized fields $L_{a b}$ satisfying the field equations (37) and (38) on $M$ are necessarily symmetric. Furthermore, taking the "dot" of $L$ (the trace of $L_{a b}$ ) and using (39b) we obtain

$$
\dot{L}=0 \text {. }
$$

Thus, there is no dynamical information in the trace of $L_{a b}$ either. One might therefore expect that the trace can be gauged away.

To see if this expectation is correct, let us analyze the restricted gauge freedom. Note first that since we had set ${ }^{+} A_{a}$ equal to zero to obtain (34) and (35), the "internal gauge" is completely fixed: we are not free to make local rotation of triads. Secondly, since we have fixed the foliation and the vector field $t^{a}$, we are now free to make only those diffeomorphisms that leave this structure invariant. These diffeomorphisms are generated by vector fields $\lambda^{a}$ on $M$ satisfying $\eta_{a b} t^{a} \lambda^{b}=0$, and $\mathscr{L}_{\lambda} t^{a}=0$. Under infinitesimal motions generated by these vector fields, the background triad changes by $V^{a}{ }_{\mathbf{i}} \rightarrow V^{a}{ }_{\mathbf{i}}+\mathscr{L}_{\lambda} V^{a}$, whence, $L_{\mathbf{i}}^{a} \rightarrow L_{\mathbf{i}}^{a}-\mathscr{L}_{\lambda} V_{\mathbf{i}}{ }^{a}$ is a gauge transformation. [This is analogous to the fact that, since $\eta_{a b}$ changes by $\eta_{a b} \rightarrow \eta_{a b}+2 \partial_{(a} \lambda_{b)}$ under infinitesimal diffeomorphism generated by $\lambda^{a}, \gamma_{a b} \rightarrow \gamma_{a b}$ $-2 \partial_{(a} \lambda_{b)}$ is a gauge transformation on linearized metrics $\gamma_{a b}$.] Contracting with $V_{b}^{\mathbf{i}}$ one obtains an equivalent form of the gauge transformations:

$$
L_{a b} \rightarrow L_{a b}+\partial_{b} \lambda_{a}
$$

with $\lambda_{a} t^{a}=0$ and $\mathscr{L}_{t} \lambda^{a}=0$.

Finally, the requirement of compatibility with the previously chosen gauge restricts the choice of permissible $\lambda^{a}$. Under (41a), the vanishing of $L_{[a b]}$ must be presented. The restricted freedom is given by (41a) with

$$
\lambda_{a}=\partial_{a} f, \text { where } \mathscr{L}_{t} f=0 .
$$

\footnotetext{
${ }^{4}$ It is helpful to express $L_{[a b]}$ as $L_{[a b]}=2 \partial_{[a} L_{b]}+\varepsilon_{a b c} \partial^{c} f$, for some one form $L_{b}$ and function $f$
} 
This freedom can be now exhausted by demanding that $L_{a b}$ be trace-free. Equation (37) now implies that it is also transverse:

$$
\partial^{a} L_{a b}=0,
$$

and (38) implies that it satisfies the evolution equation:

$$
\dot{L}_{b}^{a}=2 \varepsilon^{a m n} \partial_{m} L_{n b} .
$$

Finally, it follows from (3") and our choice of $\underset{\sim}{N}(s)$ and $t^{a}(s)$ that the linearized metric is given by $2 L_{a b}$. Thus, the linearization of our self-dual framework in an appropriate gauge yields linearized metrics which are symmetric, traceless, transverse and which satisfy the first order evolution equation (43). Apart from convention dependent numerical coefficients, this is precisely the standard characterization of weak, right circularly polarized gravitational waves.

\section{Discussion}

The notion of half-flat metrics refers only to 4-dimensional fields. A priori, therefore, it is surprising that a $3+1$ decomposition (using appropriate fields) simplifies the field equations so dramatically. The simplicity leads one to conjecture that our triad, $V^{a}$, of vector fields may in fact be closely related to some intrinsic mathematical structures that manifolds with half-flat metrics naturally admit. Recently, Robinson [9] has shown that this is indeed the case. Let us begin with a summary of his results. For simplicity, let us restrict ourselves to real, Riemannian half-flat metrics. Then, it is well-known [10] that a manifold $M$ admits a half-flat metric $g_{a b}$ if and only if it is hyperkähler, i.e., if and only if it admits three distinct integrable, almost complex structures, $J_{\mathbf{i} b}^{a}, \mathbf{i}=1,2,3$, compatible with the metric $g_{a b}$, satisfying the quaternionic relation

$$
J_{\mathbf{i}} J_{\mathbf{j}}=\varepsilon_{\mathbf{i j k}} J_{\mathbf{k}}-\delta_{\mathbf{i j}} .
$$

Thus, a half-flat space $\left(M, g_{a b}\right)$ admits three independent Kähler structures. Furthermore, the Kähler forms, $J_{\mathbf{i} a b}:=J_{\mathbf{i} a}{ }^{m} g_{m b}$, are themselves (anti-) self dual if $g_{a b}$ is itself (anti-) self dual. Therefore, given a foliation of $M, J_{\mathbf{i} a b}$ are completely determined by their "electric parts," $E_{\mathbf{i} a}:=J_{\mathbf{i} a b} \zeta^{b}$, with respect to the unit normal $\zeta^{b}$ to the leaves of the foliation. Thus, the information about the hyperkähler structure can be coded in a spatial, orthonormal triad $E_{\mathbf{i} a}$. Somewhat surprisingly, however, it turns out that this natural orthonormal triad does not satisfy simple differential equations. But if one scales all the triad vectors by a suitable scalar density, the resulting orthogonal triad $V^{a}{ }_{i}$ satisfies precisely our Eqs. (1) and (2)! (Recall that our triad $V^{a}$ is orthogonal but not orthonormal with respect to $g_{a b}$.) Thus, up to normalization, our triads do have an intrinsic geometric meaning. To obtain analytically simple differential equations on geometrically natural structures, however, two unexpected steps are necessary: the introduction of a foliation and scaling of the natural orthonormal triad on each leaf of foliation.

The interplay between hyperkähler structures and the variables used in this paper suggests certain generalizations. First, since the notion of half-flatness admits an extension to higher dimensions in terms of hyperkähler structures [11], our characterization may also do so, thereby simplifying the analogous higher 
dimensional equations. Second, in 4-dimensions, there is an interplay between Einstein spaces and quaternionic manifolds, i.e. manifolds that admit three independent integrable almost complex structures, $J_{\mathbf{i} a}{ }^{b}$, which satisfy ${ }^{4} D_{a} J_{\mathbf{i} b}{ }^{c}$ $=\left(T_{a \mathbf{i}}{ }^{\mathbf{k}}\right) J_{\mathbf{k} b}{ }^{c}$ (rather than ${ }^{4} D_{a} J_{\mathbf{i} b}{ }^{c}=0$ as in the hyperkähler case.) On the other hand, Ashtekar and Renteln have shown that variables $\left(\tilde{\sigma}^{a},{ }^{ \pm} A_{a}\right)$ can be used to obtain, in a simple way, a large class of Einstein spaces with (anti-) self dual Weyl curvature (see e.g. [12]). It is quite possible that, by analyzing the relation between the hyperquaternionic structures and $\left(\tilde{\sigma}^{a},{ }^{ \pm} A_{a}\right)$, one can show that all Einstein spaces with self dual Weyl curvature can be obtained by the simple ansatz given in [12].

Let us return to the 4-dimensional half-flat case treated in this paper. The reduced form, (1) and (2), of half-flat equations is simple and geometric. Equation (2) in particular resembles Euler's equations for a rigid body. The rich literature on generalizations of Euler's equations to infinite dimensional systems suggests that (1) and (2) may be exactly integrable. In any case, these equations - unlike those that naturally arise in the $H$-space and twistor programs - are in a form that is well suited for a standard Hamiltonian treatment. It would be nice to cast them in a Hamiltonian framework since self dual systems do not normally admit an intrinsic phase-space description: in the Yang-Mills case, for example, the pull-back of the symplectic structure to the self dual part of the phase-space (of all complex YangMills fields) is totally degenerate and the restriction of the Hamiltonian, identically zero.

Acknowledgements. We are grateful to David Robinson for telling us about quaternionic and hyperkähler manifolds. Most of the material in Sect. 6 is a result of discussions with him. This work was supported in part by NSF grants PHY 82-17853, PHY 82-01094, PHY 85-06686, PHY 03072, and PHY 86-12424, supplemented by funds from NASA, and by a grant from the Research Corporation.

\section{References}

1. Ko, M., Ludvigsen, M., Newman, E.T., Tod, K.P.: Phys. Rep. 71, 51 (1981)

2. Ludvigsen, M., Newman, E.T., Tod, K.P.: J. Math. Phys. 22, 818 (1981)

3. Penrose, R.: Gen. Rel. Grav. 7, 31 (1976)

4. Sen, A.: J. Math. Phys. 22, 1718 (1981); Int. J. Theor. Phys. 21, 1 (1981)

5. Ashtekar, A.: Physica 121A, 51 (1984)

6. Ashtekar, A.: A new Hamiltonian formulation of general relativity. Phys. Rev. D 36, 1587 (1987)

7. Penrose, R., Rindler, W.: Spinors and space-time. London: Cambridge University Press 1984

8. Penrose, R.: In: Advances in twistor theory. Hughston, L.P., Ward, R.S. (eds.). San Francisco: Pitman 1979

9. Robinson, D.C.: In: New perspectives on canonical gravity. Ashtekar, A. (ed.). Naples: Bibliopolis (in press)

10. Yano, K., Kon, M.: Structures on manifolds. Singapore: World Scientific 1984 Besse, A.L.: Einstein manifolds. Berlin, Heidelberg, New York: Springer 1987

11. Hitchin, N.J., Karlhede, A., Lindström, U., Rocek, M.: Commun. Math. Phys. 108, 535 (1987)

12. Ashtekar, A.: In: Contemporary mathematics. Isenberg, J. (ed.). Providence: American Mathematical Society 1987

Communicated by S.-T. Yau

Received August 10, 1987; in revised form November 6, 1987 